\section{ORIGINAL RESEARCH}

T.T. Le

N.J. Fischbein

J.B. André

C. Wijman

J. Rosenberg

G. Zaharchuk

\title{
Identification of Venous Signal on Arterial Spin Labeling Improves Diagnosis of Dural Arteriovenous Fistulas and Small Arteriovenous Malformations
}

BACKGROUND AND PURPOSE: DAVFs and small AVMs are difficult to detect on conventional MR imaging/MRA or CTA examinations and often require DSA for definitive diagnosis. The purpose of this study was to assess the value of venous signal intensity on ASL imaging for making this diagnosis.

\begin{abstract}
MATERIALS AND METHODS: Two neuroradiologists and 1 neurologist reviewed MR imaging studies in 26 patients, 15 of whom had DSA-proved DAVFs or small $(<2 \mathrm{~cm})$ AVMs. Pseudocontinuous ASL was performed at 1.5T with background-suppressed 3D-FSE readout. Using a 5-point scale, these readers assessed the likelihood of positive findings on a DSA study before and after reviewing the ASL findings. Agreement on imaging findings, including venous ASL signal intensity, was performed by using $\kappa$ statistics. Logistic regression and ROC analysis were performed to determine which imaging findings improved diagnosis.
\end{abstract}

RESULTS: Venous ASL signal intensity was seen frequently in cases with positive findings on DSA. The sensitivity and specificity of venous ASL signal intensity for predicting positive findings on a DSA study were $78 \%$ and $85 \%$, respectively. On ROC analysis, there was a significant increase in the AUC after review of the $A S L$ images $(A \cup C=0.798$ pre-ASL, $A \cup C=0.891$ post-ASL; $P=.02$ ). Multivariate regression identified venous $A S L$ signal intensity as the strongest predictor of positive findings on a DSA study, with an odds ratio of $17.3(95 \% \mathrm{Cl}, 3.3-90.4)$.

CONCLUSIONS: Identifying venous ASL signal intensity improved detection of DAVFs and small AVMs. Attention to this finding may improve triage to DSA in patients with suspected small vascular malformations.

ABBREVIATIONS: $\mathrm{ASL}=$ arterial spin labeling; $\mathrm{AUC}=$ area under the $\mathrm{ROC}$ curve; $\mathrm{Cl}=$ confidence interval; DAVF = dural arteriovenous fistula; $I \mathrm{CH}=$ intracranial hemorrhage; $\mathrm{PCASL}=$ pseudocontinuous ASL; PLD = postlabeling delay; $\mathrm{ROC}=$ receiver operating characteristic

D AVFs and AVMs are cerebral vascular malformations characterized by arteriovenous shunting, with direct communication between the arterial and venous circulations without an intervening capillary bed. The criterion standard for diagnosis of such vascular malformations has traditionally been DSA, which permits visualization of the arterial feeders, fistula site, and venous drainage with high spatial and temporal resolution.

ASL is an emerging noncontrast MR imaging technique in which arterial blood water is electromagnetically labeled proximal to the brain and used as a diffusible flow tracer. ${ }^{1}$ Under normal conditions, most labeled water is extracted at the capillary level into the tissue, giving rise to the parenchymal perfusion ASL signal intensity. Because the T1 decay of the labeled water is short compared with capillary

Received March 31, 2011; accepted after revision May 18.

From the Departments of Radiology (T.T.L., N.J.F., J.B.A., J.R., G.Z.) and Neurology and Neurological Sciences (C.W.), Stanford University and Stanford University Medical Center, Stanford, California.

Please address correspondence to Greg Zaharchuk, MD, PhD, 1201 Welch Rd, PS-04, Stanford University Medical Center, Mail code 5488, Stanford, CA 94305-5488; e-mail: gregz@stanford.edu

\footnotetext{
EE Indicates article with supplemental on-line table.

Indicates article with supplemental on-line figure.

http://dx.doi.org/10.3174/ajnr.A2761
}

transit time, ASL signal intensity is not typically seen within intracerebral veins. Because DAVFs and AVMs lack a capillary bed, there is no water extraction and the transit time of the labeled water is shortened; these factors can result in venous ASL signal intensity. ${ }^{2-4}$

Large DAVFs and AVMs are usually easily visible on CT/ CTA and/or conventional MR imaging/MRA and do not present a significant diagnostic challenge. MR imaging signs of smaller DAVFs or AVMs span a wide spectrum, however, ranging from no demonstrable lesion to nonspecific findings such as ICH, white matter hyperintensity, and edema to more specific findings such as dilated serpiginous vessels and venous pouches. Especially in the presence of edema and mass effect from ICH, small vascular malformations remain difficult to detect with standard MR imaging methods. ${ }^{5}$ While newer techniques, such as time-resolved MRA, ${ }^{6-8}$ are becoming available and may allow increased sensitivity to small lesions, most patients still require an invasive DSA study for definitive diagnosis. The ability to identify small vascular shunts with a noninvasive technique should allow better triage to confirmatory DSA and enable joint confirmatory and therapeutic angiography in a single session, thus reducing cost and risk to the patient. The purpose of this study was to assess the added value of venous ASL signal intensity to detect small AVMs and DAVFs. 


\section{Materials and Methods}

\section{Patient Population}

This retrospective study was approved by our institutional review board. Review of our Neurointerventional Radiology data base yielded 54 patients with DAVFs or small AVMs (defined for the purpose of this study as $<2 \mathrm{~cm}$ ) diagnosed at our institution between September 2007 and September 2010. From this group, 15 patients ( 8 DAVFs and 7 small AVMs) also underwent MR imaging that included ASL (On-line Table 1). Within this study group, 2 patients had residual AVMs and 1 patient had a residual DAVF following embolization or surgery. Within the study group, 9 patients had intraparenchymal hemorrhage, 3 patients had subarachnoid hemorrhage, 1 patient presented with seizure, 1 patient was found to have an AVM on a follow-up study for Moyamoya disease, and 1 patient was found to have a DAVF on a follow-up study for carotid cavernous fistula. The control group consisted of 11 patients who met the following criteria: the same time period, the presence of ICH (4 patients had intraparenchymal hemorrhage and 7 patients had subarachnoid hemorrhage), a suspected vascular lesion, negative findings on a DSA study, and an MR imaging study including ASL. The age and sex breakdown of the 2 groups was as follows: study group: mean age, 58 years; range, 11-69 years; male/female ratio, 9:6; control group: mean age, 49 years; range, 20-75 years; male/female ratio, 5:6. Presenting symptoms were similar and included headache in 7 patients, tinnitus in 1 patient, unilateral weakness in 12 patients, and seizure in 1 patient; 18 patients had ICH. The interval between the initial DSA and MR imaging examinations ranged from 0 to 5 days. Two patients with positive findings ( 1 with a DAVF [patient 7] and 1 with an AVM [patient 5]) had initial DSA examinations read as having negative findings, presumably due to obscuration of the vascular malformation from mass effect due to ICH; follow-up DSA studies 7 and 28 days later, respectively, had positive findings.

\section{Imaging Methods}

All imaging was performed at 1.5T (Signa; GE Healthcare, Milwaukee, Wisconsin) and included the following sequences: 3D time-offlight MRA (TR/TE, 34/3.1 ms; FOV, 24 cm; matrix, $512 \times 128$; 1-mm thick sections), axial diffusion-weighted (TR/TE, 6000/70 ms; $b=1000 \mathrm{~s} / \mathrm{mm}^{2}$ ), axial gradient-echo (TR/TE, 600/30 ms), T2weighted FSE (TR/TE, 4717/85 ms), axial fluid-attenuated inversion recovery (TR/TE/TI, 8802/110/2200 ms), and T1 postcontrast (TR/TE 600/15 ms). All of the anatomic imaging was performed with 5-mm section thickness, 1.5-mm skip, and 24-cm FOV. pcASL imaging (TR/TE, 5500/2.5 ms) was performed by using a labeling period of $1500 \mathrm{~ms}$ followed by a PLD of $2000 \mathrm{~ms},{ }^{9}$ with the labeling plane at the level of the foramen magnum. Readout was performed with 3D background-suppressed FSE, with an in-plane and through-plane spatial resolution of 3 and $4 \mathrm{~mm}$, respectively.

All patients underwent catheter-based DSA as part of their clinical work-up for ICH of unclear origin or suspicion of a vascular malformation in dedicated biplane neuroangiography suites (Axiom Artis; Siemens, Erlangen, Germany). DSA examinations included anteroposterior and lateral views with selective injection of the appropriate internal carotid, external carotid, and/or vertebral artery with iodinated contrast medium (iohexol, Omnipaque 300; GE Healthcare, Chalfont St. Giles, United Kingdom).

\section{Radiologic Assessment}

The MR imaging examinations were independently reviewed by 2 neuroradiologists and 1 neurologist blinded to patient history and DSA results. If there was an initial noncontrast CT examination, the readers were allowed to review it, because this was thought to most closely approximate clinical practice. Initial scoring was performed with access to all of the MR imaging sequences except the ASL study. The reviewers first recorded the presence of ICH (including hemosiderin staining), edema, abnormal MRA, serpiginous vessels, and abnormal venous enhancement (in either a venous pouch or prominent draining vein on postcontrast imaging). The reviewers then stated whether they thought there was a DAVF or AVM, by using the following 5-point scale: 1) very unlikely, 2) unlikely, 3) equally likely and unlikely (neutral), 4) likely, and 5) very likely. After this decision, they then reviewed the ASL imaging of the same patient and additionally determined the presence of venous ASL signal intensity, defined as either high signal intensity in the location of a major venous structure (superior sagittal sinus, transverse sinus, sigmoid sinus, straight sinus, vein of Galen, internal cerebral veins) or in cortical veins. It was possible to cross-reference the ASL images to the anatomic imaging to aid in the assessment. Finally, the readers were again asked to grade the likelihood of a DAVF or small AVM by using the same scale. Independent of these 3 reviewers, 2 other neuroradiologists evaluated the ASL images with direct comparison with the angiographic results. The presence of a DAVF or AVM on DSA was considered the criterion standard and was based on the DSA clinical report.

\section{Statistical Analysis}

Interobserver agreement for imaging findings among the 3 readers was assessed with the $\kappa$ statistic. Sensitivity and specificity were assessed by means of univariate and multivariate logistic regressions of DSA results on imaging features. Assessment of the contribution of the venous ASL signal-intensity feature was done by a likelihood ratio test between multivariate models with and without the feature.

To test the added value of ASL, we performed sensitivity, specificity, and accuracy of reader assessments with and without the use of venous ASL signal-intensity information with ROC analysis, by nonparametric estimation of the AUC. Comparison of pre- and post-ASL assessment AUCs was done with the DeLong algorithm.

\section{Results}

Venous ASL signal intensity was frequently seen in cases that were deemed positive on DSA study (Figs 1-3 and 6). Table 1 shows the $\kappa$ values for agreement of the 3 readers overall and for specific imaging features. Agreement was moderate-togood, and highest for the presence of venous ASL signal intensity $(\kappa=0.64 ; 95 \% \mathrm{CI}, 0.40-0.85)$. The sensitivity, specificity, positive predictive value, and negative predictive value of venous ASL signal intensity to predict a positive DSA study were 78\% (95\% CI, 63\%-89\%), 85\% (95\% CI, 68\%-95\%), 88\% (95\% CI, 73\%-96\%), and 74\% (95\% CI, 57\%-87\%), respectively.

Figure 4 is a histogram of pooled score frequency pre- and post-ASL review for DSA cases with positive and negative findings. The effect of the ASL information was to move readers' assessments to the ends of the 5-point scale, regardless of the presence or absence of a vascular lesion $(P<.0001)$. The pooled post-ASL assessments had a significantly $(P=.02)$ higher AUC than the pre-ASL assessments: pre-ASL, 0.798 (95\% CI, 0.707-0.890); post-ASL, 0.891 (95\% CI, 0.819- 



Fig 1. Patient 12. A 64-year-old man presenting with complex partial seizure, with electroencephalography localized to the left temporal lobe. $A$, Axial gradient recalled-echo image shows hemosiderin staining in the left temporo-occipital cortex and along the tentorium cerebelli. $B$, Axial T1-weighted postcontrast image shows mildly prominent serpiginous vasculature in this region. $C$, Axial ASL perfusion shows high-signal-intensity labeled spins within the venous confluence and left transverse and sigmoid sinuses, indicating the presence of venous shunting surgery. $D$, Lateral DSA left external carotid artery injection, shows a left transverse sinus DAVF with arterial feeding from the left middle meningeal artery and, to a lesser extent, from the left occipital artery. Venous drainage is to the left transverse and sigmoid sinuses and left internal jugular vein.

0.962) (Figure 5). The post-ASL ROC curve also showed improved symmetry, suggesting that the MR imaging examination including ASL became a more well-rounded predictor, primarily by increasing sensitivity.

Table 2 shows the results of univariate and multivariate logistic regression of the individual imaging features in predicting positive findings on a DSA study. Univariate regressions showed that lack of edema, the presence of abnormal MRA findings, serpiginous vessels, venous enhancement, and venous ASL signal intensity were significant predictors of positive findings on a DSA study. Due to the high correlations between abnormal MRA findings and serpiginous vessels (tetrachoric $r=0.77$ ) and between venous enhancement and serpiginous vessels $(r=0.67)$, significant independent predictors in a multivariate regression were only lack of edema, the presence of serpiginous vessels, and venous ASL signal intensity. The venous ASL signal intensity had the highest odds ratio for prediction (17.3; 95\% CI, 3.3-90). The inclusion of venous ASL significantly improved the fit of the multivariate regression compared with using only the other 5 features $(P=.0001$ by likelihood-ratio test). On the basis of the multivariate results, we tested the ROCs of a score consisting of 1 point each for the following: 1) absence of edema, 2) serpiginous vessels, and 3 ) venous ASL signal intensity. The AUC of this simplified score was 0.902 (95\% CI, 0.84-0.97). If the MR imaging examination received a score of $\geq 2$, the sensitivity, specificity, positive predictive value, and negative predictive value to predict a DSA study with positive findings were $76 \%(95 \% \mathrm{CI}$, 61\%-87\%), 97\% (95\% CI, 84\%-100\%), 97\% (95\% CI, 85\%$100 \%$ ), and $74 \%$ (95\% CI, 59\%-87\%), respectively.

Before evaluating the ASL sequence, readers scored $21 \%$ of the studies as "neutral" (score of 3 ) regarding the presence of vascular malformation (16/78 instances). These are cases in which triage to DSA might be debated. Seven of 16 of these $(44 \%)$ were in patients with positive findings on DSA, and in all of these cases, the readers thought that a vascular malformation was more likely on post-ASL review (5 were scored as likely and 2 were scored as very likely). In the 9 cases with negative findings on DSA, 6 were correctly predicted as unlikely ( 2 as unlikely and 4 as very unlikely), while 3 were incorrectly thought to be more likely (all scored likely). In 2 of these cases, the readers thought that venous ASL signal intensity was present. After ASL review, the number of cases graded as neutral dropped from 16 to 3 , indicating an increase in confidence of diagnosis.

Post hoc review of the MR imaging with access to the DSA 

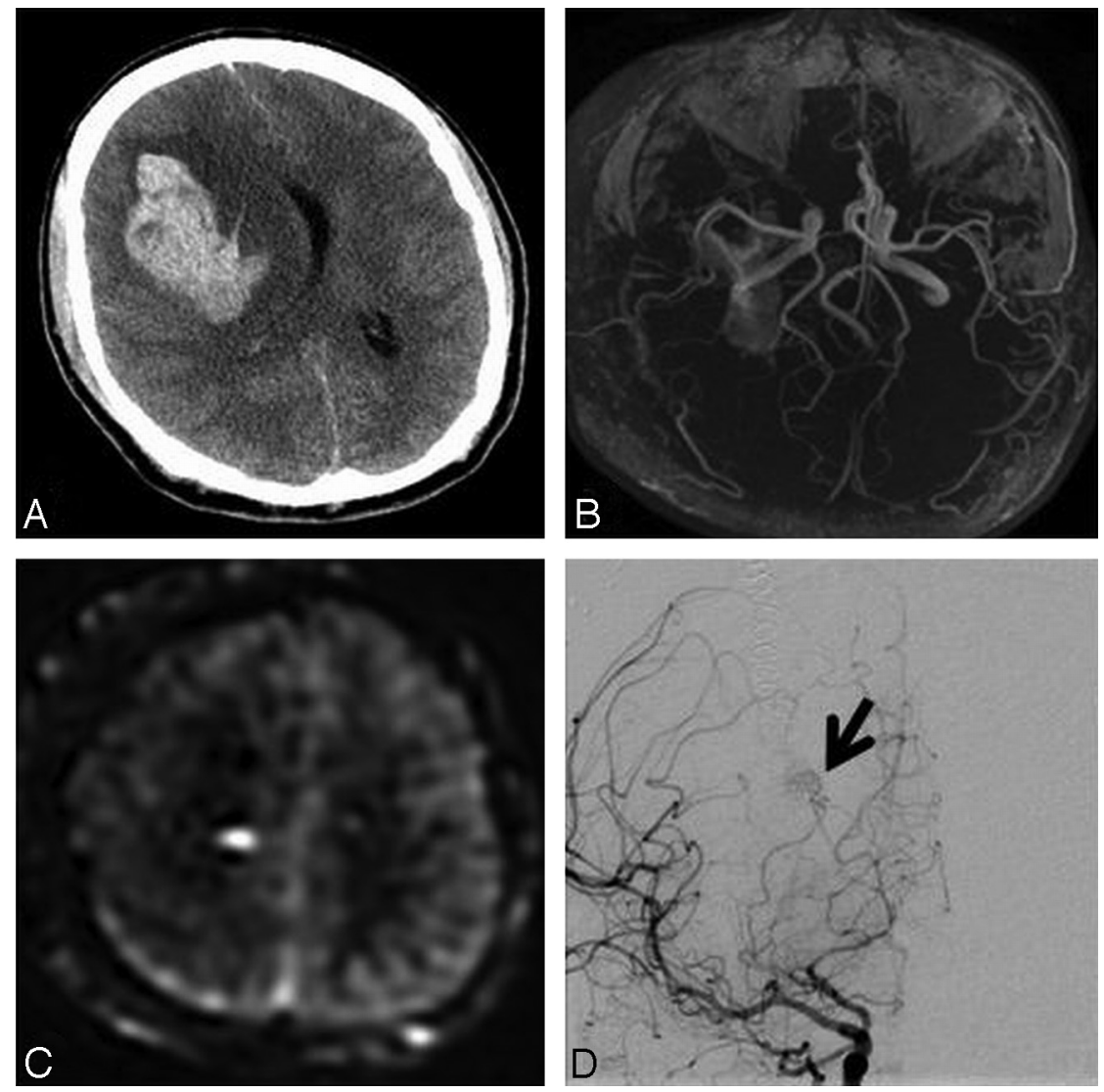

Fig 2. Patient 5. A 25-year-old man with sudden onset of headache while weightlifting, followed by confusion and loss of consciousness. $A$, Axial noncontrast CT scan shows a large right frontal hematoma with significant edema, mass effect, and brain herniation. The patient was immediately taken to the operating room for right frontal craniotomy decompression. $B$, Postdecompression time-of-flight MRA findings are normal, without MR imaging evidence of AVM or DAVF. C, Axial ASL image shows focal high signal intensity at the medial right frontal lobe (white arrow), concerning for the presence of a shunt lesion. Initial angiogram (not shown) obtained on the same day as the MR imaging study failed to demonstrate the presence of an AVM, likely due to edema and mass effect. $D$, Repeat angiogram after 4 weeks shows a small $(<1 \mathrm{~cm})$ right frontal lobe AVM (black arrow). The feeding vessel is the right anterior choroidal artery, with deep venous drainage into the right internal cerebral vein.

findings demonstrated the reasons for some of the discrepancies of the blinded readers. In some cases, ASL signal intensity in the callosomarginal branch of the anterior cerebral artery (representing slow arterial flow) was attributed to a venous structure (the superior sagittal sinus) (On-Line Fig 1). Another error was caused by the presence of a small amount of focal ASL signal intensity in an unexpected region-for example, in the case of a patient with a very slow-flow left petrous apex DAVF (Fig 6).

\section{Discussion}

This study demonstrates that identifying ASL signal intensity within intracerebral veins on pcASL improves the diagnostic ability of a broad range of readers to detect small AVMs and DAVFs. Recent reports have suggested that other advanced imaging techniques are capable of detecting and evaluating such lesions, including 4D contrast-enhanced MRA, ${ }^{6-8,10,11}$ dynamic MRA, ${ }^{5}$ phase-contrast MRA, ${ }^{12}$ and MR bolus perfusion-weighted imaging. ${ }^{13}$ Relatively few studies have been reported on the possible value of ASL imaging. ${ }^{2-4,13,14}$ Most of these have endeavored to quantitate flow to the nidus or the perinidal region, which is challenging due to the retention of labeled water within vessels rather than within tissue and which we did not attempt. None evaluated whether ASL is of clinical value in detecting otherwise occult lesions. The cur- rent report shows that identifying ASL signal intensity in the venous system can be a sign of small shunting vascular malformations, and incorporating this information leads to a better clinical assessment. This is important, because while angiography in proper hands is not a high-risk procedure, it is time- and personnel-intensive and can cause patient discomfort. Even following DSA, mass effect from ICH and edema can obscure small lesions, and repeat DSA is generally recommended to increase detection sensitivity, as occurred in 2 cases in our series.

ASL is becoming more common in the clinical setting due to recent technical advances, including the use of spin-echo readout and background suppression. ${ }^{15}$ Other variations of ASL, however, which use 2D echo-planar imaging have artifacts caused by ICH or embolic material. For example, a prior report evaluating time-resolved imaging of contrast kinetics (TRICKS) and ASL in a DAVF found that ASL was often uninterpretable due to motion and/or $\mathrm{ICH}^{3}$ We suspect that the higher signal intensity-to-noise ratio, reduced susceptibility artifacts, decreased motion sensitivity due to background suppression, and 3D FSE readout of the current ASL sequence all contributed to its value. All ASL images in this study were interpretable, including in patients with embolic material and large hematomas, and there was good inter-reader agreement regarding venous ASL signal intensity. 

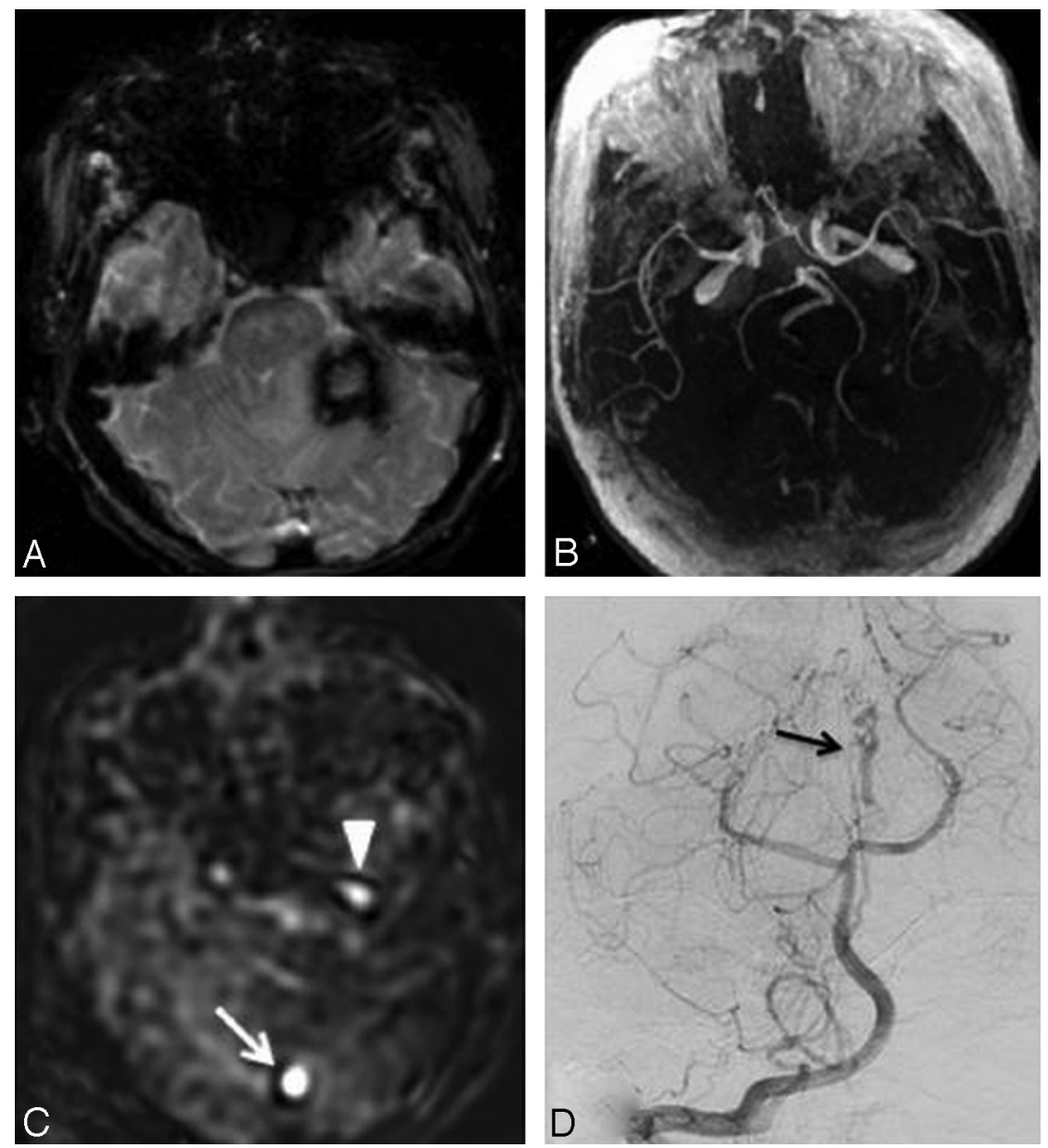

Fig 3. Patient 2. A 76-year-old man presenting with worsening headache and vomiting. A, Axial gradient recalled-echo MR image shows a left cerebellar hemorrhage. $B$, MRA does not reveal an obvious vascular lesion. $C$, ASL image shows high signal intensity within the venous confluence (white arrow) and left internal jugular vein (white arrowhead), suspicious for a shunt lesion. D, Frontal DSA confirms a small AVM, fed by a small branch of the left vertebral artery; venous drainage is to the venous confluence, left transverse sinus, and left internal jugular vein.

\begin{tabular}{lcc}
\hline $\begin{array}{l}\text { Table 1: Agreement of the } \mathbf{3} \text { readers overall and with respect to } \\
\text { individual features }\end{array}$ \\
\hline Readers $^{\text {a }}$ & $\kappa$ & $95 \% \mathrm{Cl}$ \\
\hline 1 and 2 & 0.45 & $0.23-.61$ \\
1 and 3 & 0.42 & $0.18-.62$ \\
2 and 3 & 0.72 & $0.47-.89$ \\
Signs & & \\
ICH & 0.30 & $-0.11-.74$ \\
Edema & 0.54 & $0.28-.74$ \\
Abnormal MRA & 0.43 & $0.17-.68$ \\
Serpiginous vessels & 0.58 & $0.32-.80$ \\
Venous enhancement & 0.29 & $0.02-.54$ \\
Venous ASL signal & 0.64 & $0.40-.85$ \\
\hline
\end{tabular}

${ }^{a}$ Reader 1 is a neurologist; readers 2 and 3 are neuroradiologists.

ASL has previously been shown to demonstrate conspicuous findings in large AVMs. ${ }^{2,3}$ ASL uses labeled water as a diffusible tracer, which is $90 \%$ extracted on the first pass through the capillary bed. Because the mean dwell time within the voxel for extravascular water is much longer than the label T1, ASL signal intensity is not typically seen in the venous system. This would not be true for some other ASL implementations, such as fluid-attenuated inversion recovery, in which veins and arteries are labeled. ${ }^{14}$ Therefore, by using pcASL, one does not see venous ASL signal intensity except in the presence of a direct arteriovenous connection, such as in AVMs and DAVFs. The magnitude of the venous ASL signal intensity depends on the number of spins traversing the fistula and the precise timing (both labeling time and PLD) as well as whether vascular crusher gradients are used. In our experience by using nonvascular-suppressed pcASL routinely, we rarely see venous ASL signal intensity, except in the setting of arteriovenous shunt lesions. In theory, one might expect to see venous ASL signal intensity in very vascular brain tumors (such as hemangioblastoma) or in subacute stroke (in which arteriovenous shunting has been described).

This study was designed to identify the added clinical value of ASL for the diagnosis of small AVMs or DAVFs. We excluded AVMs $>2 \mathrm{~cm}$ to better capture patients in whom diagnosis is challenging on noninvasive imaging. ROC analysis showed that the readers performed significantly better after reviewing the ASL sequence. This was due to 2 factors: 1 ) the readers' scores increased if they identified venous ASL signal intensity, indicating that they thought a vascular malformation was more likely; and 2) the presence of venous ASL signal intensity had a high positive predictive value to detect these lesions. Both univariate and multivariate regression demonstrated that venous ASL signal intensity was associated with the highest odds ratio to predict positive findings on a DSA 


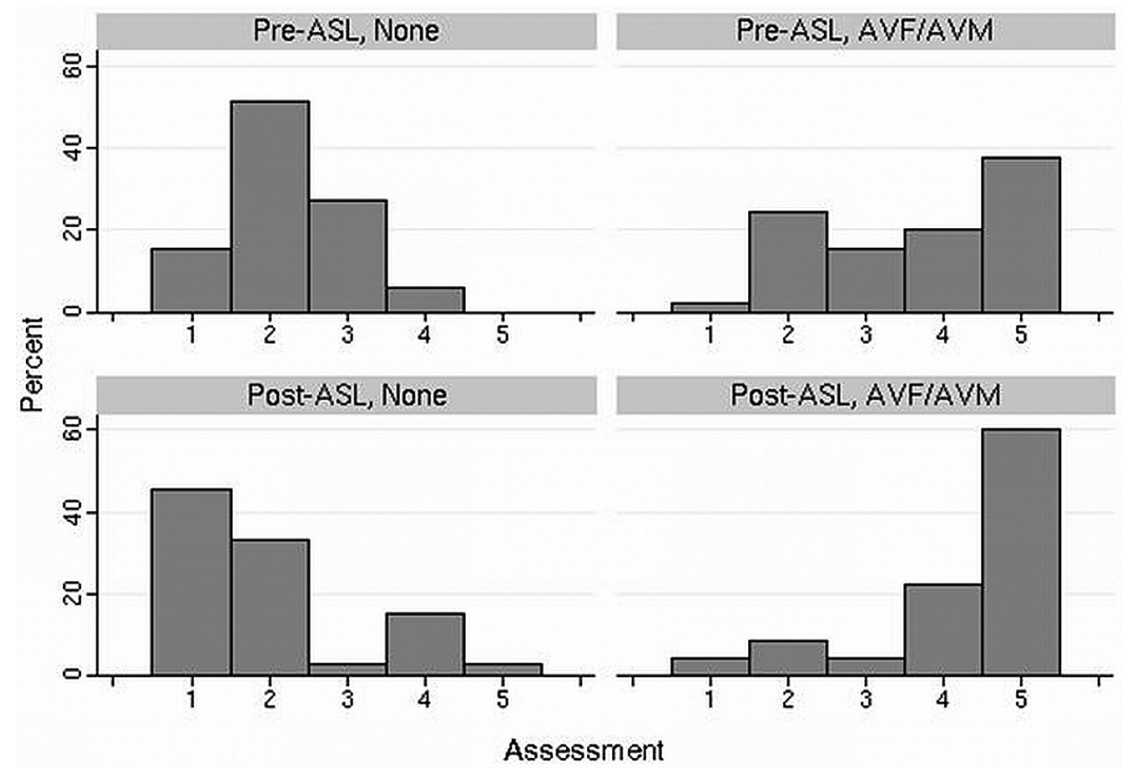

Fig 4. Histograms of pooled scores of the 3 readers regarding the likelihood of DAVF/AVM ( $1=$ very unlikely, $2=$ unlikely, $3=$ neutral, $4=$ likely, $5=$ very likely) before and after reviewing the ASL images, for cases with both negative and positive findings on DSA. Note that the distribution before reviewing ASL was skewed toward the correct diagnosis, but this effect was magnified following ASL review. In particular, the number of cases deemed "neutral" (score of 3) markedly decreased following review of ASL, suggesting that the readers had higher confidence in their decisions post-ASL.
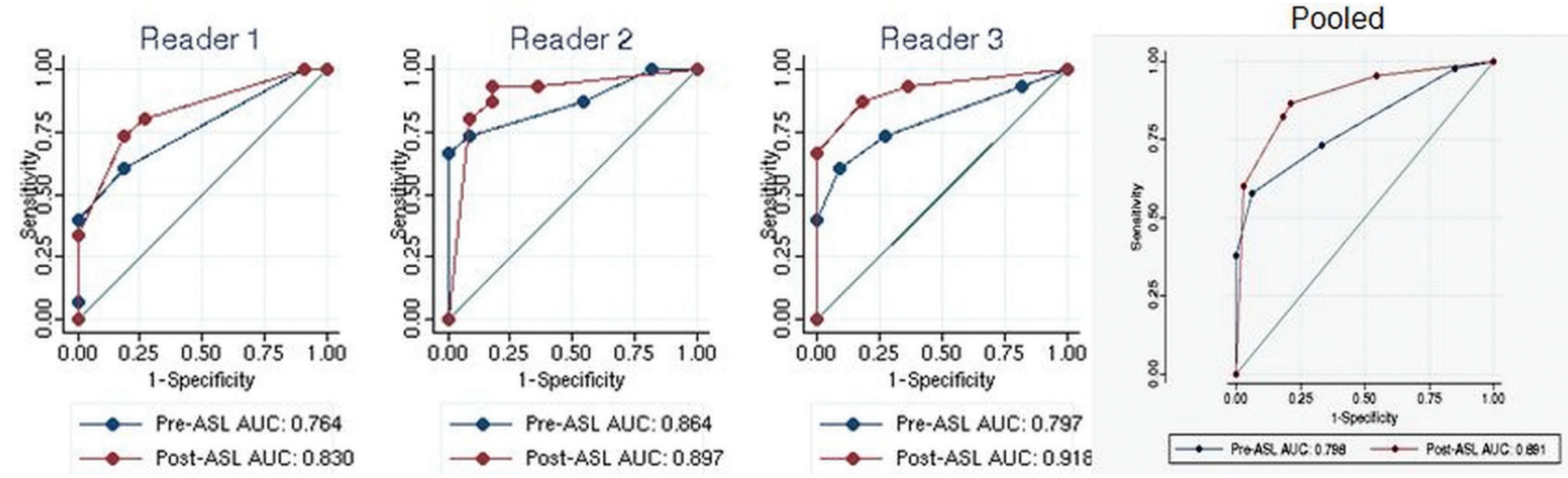

Fig 5. ROC curves for the pooled and individual readers pre- and postreview of the ASL images. The increase in the AUC was significant ( $P=.02)$. The post-ASL curve is a more well-rounded predictor and indicates that the readers' sensitivity for identifying a vascular malformation has increased after reviewing the ASL images. Reader 1 is a neurologist; readers 2 and 3 are neuroradiologists.

study. Using this information, we devised a simple scoring system incorporating 3 factors (absence of edema, presence of serpiginous vessels, and venous ASL signal intensity), which had equally good performance as the reader's judgment. Finally, we found that equivocal scores (score of 3 , neutral) decreased dramatically following ASL review and that the final judgment in such cases was overwhelmingly the correct one.

This study has several limitations. First, it was a retrospective study, and unknown biases could be present. We suspect that any biases would work against finding positive results because the cases chosen were challenging, the level of suspicion of the readers was high (as they were aware that they were to search particularly for these lesions), and the readers were highly experienced. With the setup of this study, the readers knew that a large percentage of the cases had DAVFs or small AVMs, and they would tend to be biased in favor of detecting these types of lesions. In an unselected population, it is likely the readers' sensitivity (due to pretest probability) would have been lower and it would have been easier to show a positive
Table 2: Univariate and multivariate logistic regression analysis of imaging features on DSA result

\begin{tabular}{|c|c|c|c|c|c|}
\hline Imaging Features & $\begin{array}{l}\text { Odds } \\
\text { Ratio }\end{array}$ & $\begin{array}{c}\text { Standard } \\
\text { Error }\end{array}$ & $Z$ & $P>z$ & $95 \% \mathrm{Cl}$ \\
\hline \multicolumn{6}{|l|}{ Univariate analysis } \\
\hline $\mathrm{ICH}$ & 0.17 & 0.19 & -1.62 & .105 & $0.02-1.45$ \\
\hline Edema & 0.30 & 0.15 & -2.48 & .013 & $0.12-0.78$ \\
\hline Abnormal MRA ${ }^{a}$ & 3.45 & 1.68 & 2.55 & .011 & $1.33-8.94$ \\
\hline Serpiginous vessels ${ }^{a}$ & 17.85 & 11.18 & 4.60 & $<.001$ & $5.23-60.94$ \\
\hline Venous enhancement $^{a}$ & 2.73 & 1.38 & 1.99 & .046 & $1.02-7.35$ \\
\hline Venous ASL signal $^{a}$ & 19.60 & 11.83 & 4.93 & $<.001$ & $6.00-63.97$ \\
\hline \multicolumn{6}{|l|}{ Multivariate analysis } \\
\hline $\mathrm{ICH}$ & 1.18 & 1.84 & 0.11 & .915 & $0.06-24.93$ \\
\hline Edema $^{a}$ & 0.12 & 0.11 & -2.34 & .019 & $0.02-0.71$ \\
\hline Abnormal MRA & 0.82 & 0.88 & -0.18 & .856 & $0.10-6.72$ \\
\hline Serpiginous vessels ${ }^{a}$ & 2.53 & 13.35 & 2.37 & .018 & $1.55-101.19$ \\
\hline$V_{\text {Venous enhancement }}{ }^{a}$ & 0.79 & 0.77 & -0.24 & .811 & $0.12-5.31$ \\
\hline Venous ASL signal $^{a}$ & 17.30 & 14.57 & 3.39 & .001 & 3.32-90.14 \\
\hline
\end{tabular}

a Significant at $P<.05$.

Multivariate post-ASL model is an improvement over the pre-ASL model $(P=.0001)$. 

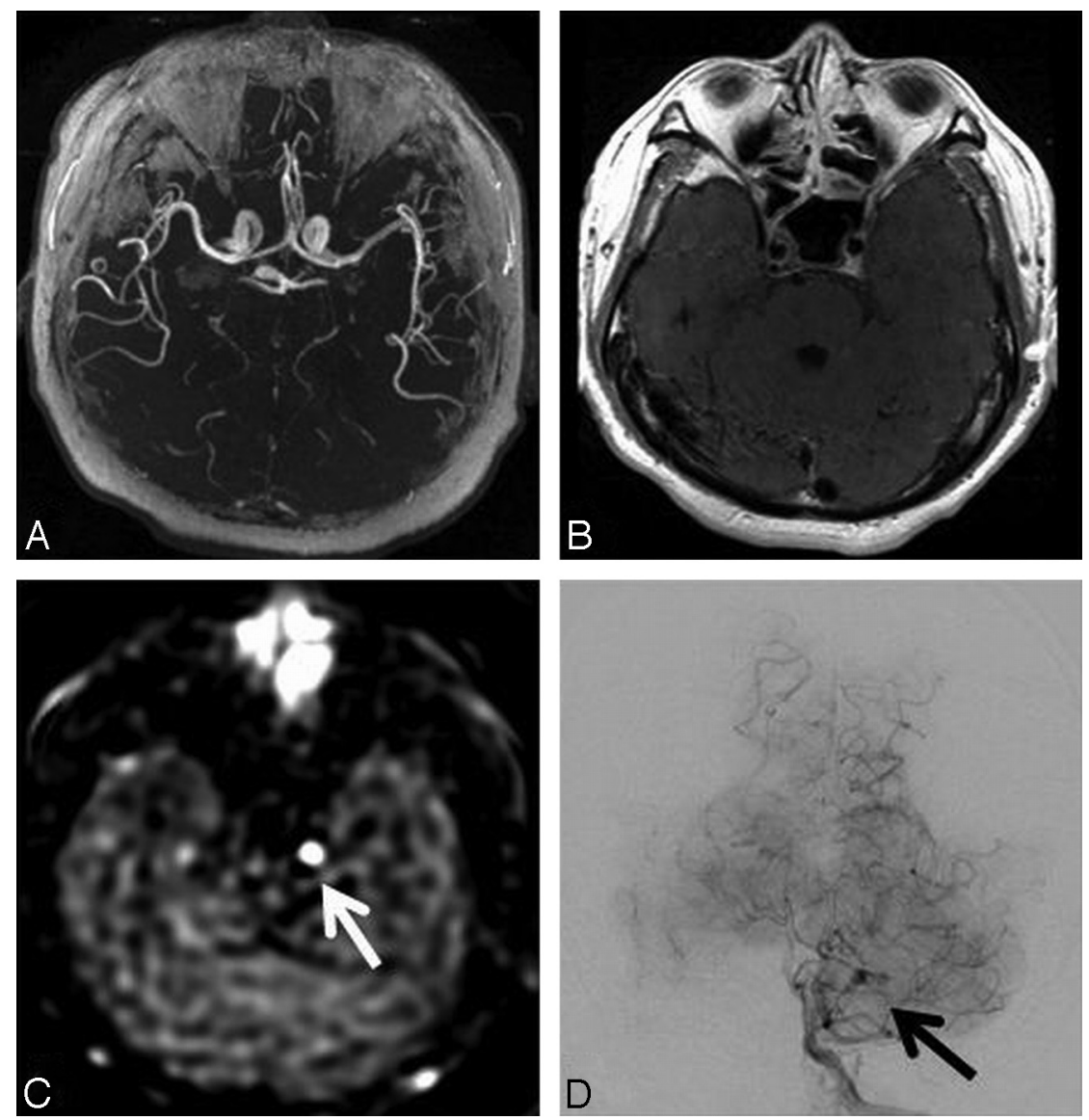

Fig 6. Patient 7. A 38-year-old man who presented to the medical center with new-onset headache. $A$ and $B$, Time-of-flight MRA image $(A)$ and axial T1-weighted postcontrast image ( $B$ show no evidence of DAVF, AVM, or aneurysm. C, ASL shows focal high signal intensity at the left petrous apex, concerning for a focal lesion (aneurysm or shunt lesion). The initial angiogram failed to demonstrate a vascular lesion (not shown). $D$, Frontal projection from the repeat angiogram (left vertebral artery injection) shows a very slow-flow left petrous apex DAVF (arrows), fed by a muscular branch of the left vertebral artery and drained via perimedullary veins. A small DAVF was confirmed at surgical resection.

effect of the ASL. Despite this, we showed that the use of ASL improved their diagnostic ability. Second, one could imagine that the cases in which ASL was acquired may differ from the general population or that cases with venous ASL signal intensity may have been more frequently triaged to DSA. We doubt this was a significant factor, given that the decision to perform ASL was often dependent on the scanner that was used, whether the sequence was operational, and whether the technologists were familiar with the sequence. Also, at the time of the study, clinicians were not routinely advised of the ASL results, so this was unlikely to have influenced the decision to request a DSA study.

Another limitation is the relatively small sample size. We only recently began routine ASL imaging in cases of ICH and suspected vascular malformation. Also, we evaluated only 1 ASL method, with a fixed labeling time and PLD and at relatively low magnetic field strength (1.5T). It is possible that this makes the results less generalizable and also that other parameters or sequences might highlight venous ASL signal intensity to a greater or lesser degree. Higher field strength will improve the overall signal intensity-to-noise ratio and should increase the conspicuity of venous ASL signal intensity. The use of vascular suppression, which is common with some implementations of ASL, might be expected to reduce sensitivity to identify venous ASL signal intensity. Also, the current ASL implementation, a 3D-FSE pcASL sequence with a very short
TE, is more suited to identifying venous ASL signal intensity compared with echo-planar imaging, which typically has longer TEs and is less sensitive to vascular labeling. Finally, we acknowledge potential problems using pooled statistics from readers with different backgrounds; we justified this because the trend of all 3 readers was similar and we wished to avoid the known problems of consensus readings.

We believe that the role of ASL in diagnosing DAVFs or small AVMs is to improve screening for these lesions by detecting venous shunting. At our institution, we currently include ASL imaging in our MR imaging protocol for $\mathrm{ICH}$ and in any cases in which a DAVF or small AVM is a concern (ie, pulsatile tinnitus). We believe that lack of venous ASL signal intensity may obviate performing DSA if our results can be validated in a larger prospective study group. Conversely, if ASL venous signal intensity is clearly identified in a patient suspected of having a DAVF or small AVM, then expedited referral to the neurointerventional service for endovascular diagnosis and treatment may be facilitated.

Disclosures: Greg Zaharchuk_UNRELATED Consultancy. GE Neuroradiology Advisory Board.

\section{References}

1. Alsop DC, Detre JA. Multisection cerebral blood flow MR imaging with continuous arterial spin labeling. Radiology 1998;208:410-16 
2. Wolf RL, Wang J, Detre JA, et al. Arteriovenous shunt visualization in arteriovenous malformations with arterial spin-labeling MR imaging. AJNR Am J Neuroradiol 2008;29:681-87

3. Kukuk GM, Hadizadeh DR, Bostrom A, et al. Cerebral arteriovenous malformations at 3.0 T: intraindividual comparative study of 4D-MRA in combination with selective arterial spin labeling and digital subtraction angiography. Invest Radiol 2010;45:126-32

4. Pollock JM, Whitlow CT, Simonds J, et al. Response of arteriovenous malformations to gamma knife therapy evaluated with pulsed arterial spin-labeling MRI perfusion. AJR Am J Roentgenol 2011;196:15-22

5. Essig M, Engenhart R, Knopp MV, et al. Cerebral arteriovenous malformations: improved nidus demarcation by means of dynamic tagging MR-angiography. Magn Reson Imaging 1996;14:227-33

6. Farb RI, Agid R, Willinsky RA, et al. Cranial dural arteriovenous fistula: diagnosis and classification with time-resolved MR angiography at 3T. AJNR Am J Neuroradiol 2009;30:1546-51

7. Nishimura S, Hirai T, Sasao A, et al. Evaluation of dural arteriovenous fistulas with $4 \mathrm{D}$ contrast-enhanced MR angiography at $3 \mathrm{~T}$. AJNR Am J Neuroradiol 2010;31:80-85

8. Hadizadeh DR, von Falkenhausen M, Gieseke J, et al. Cerebral arteriovenous malformation: Spetzler-Martin classification at subsecond-temporal-resolution four-dimensional MR angiography compared with that at DSA. Radiology 2008;246:205-13
9. Dai W, Garcia D, de Bazelaire C, et al. Continuous flow driven inversion for arterial spin labeling using pulsed radiofrequency and gradient fields. Magn Reson Med 2008;60:1488-97

10. Taschner CA, Gieseke J, Le Thuc V, et al. Intracranial arteriovenous malformation: time-resolved contrast-enhanced MR angiography with combination of parallel imaging, keyhole acquisition, and k-space sampling techniques at 1.5 T. Radiology 2008;246:871-79

11. Meckel S, Maier M, Ruiz DS, et al. MR angiography of dural arteriovenous fistulas: diagnosis and follow-up after treatment using a time-resolved 3D contrast-enhanced technique. AJNR Am J Neuroradiol 2007;28:877-84

12. Cellerini $M$, Mascalchi M, Mangiafico $S$, et al. Phase-contrast MR angiography of intracranial dural arteriovenous fistulae. Neuroradiology 1999;41:487-92

13. Noguchi K, Kuwayama N, Kubo M, et al. Intracranial dural arteriovenous fistula with retrograde cortical venous drainage: use of susceptibilityweighted imaging in combination with dynamic susceptibility contrast imaging. AJNR Am J Neuroradiol 2010;31:1903-10. Epub 2010 Sep 2

14. Noguchi K, Kuwayama N, Kubo M, et al. Flow-sensitive alternating inversion recovery (FAIR) imaging for retrograde cortical venous drainage related to intracranial dural arteriovenous fistula. Neuroradiology 2011;53:153-58. Epub 2010 Jun 20

15. Mani S, Pauly J, Conolly S, et al. Background suppression with multiple inversion recovery nulling: applications to projective angiography. Magn Reson Med 1997;37:898-905 